PROCEEDINGS OF THE

AMERICAN MATHEMATICAL SOCIETY

Volume 130, Number 2, Pages 477-482

S 0002-9939(01)06129-9

Article electronically published on September 19, 2001

\title{
THE CONVERGENCE-EXTENSION THEOREM OF NOGUCHI IN INFINITE DIMENSIONS
}

\author{
DO DUC THAI AND TRAN NGOC GIAO
}

(Communicated by Steven R. Bell)

\begin{abstract}
In this note we give generalizations of Noguchi's convergenceextension theorem to the case of infinite dimension.
\end{abstract}

\section{INTRODUCTION}

The convergence-extension theorem of Noguchi referred to in the title of this note can be stated as follows (see $\mathrm{Ko}$ or $[\mathrm{N}-\mathrm{O}]$ ):

Let $M$ be relatively compact hyperbolically imbedded into $Y$. Let $X$ be a complex manifold and $A$ a complex hypersurface of $X$ with only normal crossings.

Assume that $\left\{f_{j}: X \backslash A \rightarrow M\right\}_{j=1}^{\infty}$ is a sequence of holomorphic mappings which converges uniformly on compact subsets of $X \backslash A$ to a holomorphic mapping $f: X \backslash A \rightarrow M$.

Then $\left\{\bar{f}_{j}: X \rightarrow Y\right\}_{j=1}^{\infty}$ converges uniformly on compact subsets of $X$ to $\bar{f}$ : $X \rightarrow Y$, where $\bar{f}_{j}: X \rightarrow Y$ and $\bar{f}: X \rightarrow Y$ are holomorphic extensions of $f_{j}$ and $f$ over $X$.

The aim of this note is to give a generalization of the above-mentioned theorem of Noguchi to the infinite dimensional case, i.e., the study in the infinite dimensional case of the following problem.

Let $f_{n}: X \rightarrow Y$ and $f: X \rightarrow Y$ be holomorphic maps, where $X$ is a Banach analytic manifold and $Y$ is a Banach analytic space. Let $A$ be a complex hypersurface of $X$ such that the sequence $\left\{f_{n}\right\}_{n=1}^{\infty}$ converges to $f$ uniformly on compact subsets of $X \backslash A$. Can we conclude that $\left\{f_{n}\right\}_{n=1}^{\infty}$ converges to $f$ uniformly on compact subsets of $X$ ?

The first theorem gives a positive answer if $Y$ is a pseudoconvex Banach analytic manifold having $C^{1}$-partitions of unity and such that every holomorphic map $\varphi$ : $\mathbf{C} \rightarrow Y$ with $\varphi(\mathbf{C})$ relatively compact in $Y$ is constant.

The second statement asserts that the answer is also positive if $A$ is a complex hypersurface of $X$ with only normal crossings and $f_{n}(X \backslash A)$ and $f(X \backslash A)$ are contained in a hyperbolically imbedded subspace of $Y$. This is the exact generalization of a theorem of Noguchi.

Received by the editors June 27, 2000.

1991 Mathematics Subject Classification. Primary 32H05, 32H15; Secondary 32M05, 32M99.

Key words and phrases. Banach analytic space, complex hypersurface of a Banach analytic manifold with only normal crossings, hyperbolically imbedded Banach analytic subspace of a Banach analytic space. 
Namely, we prove the following:

Theorem A. Let $Y$ be a pseudoconvex Banach analytic manifold having $C^{1}$-partitions of unity. Suppose that every holomorphic map $\varphi: \mathbf{C} \rightarrow Y$ with $\varphi(\mathbf{C}) \subset \subset Y$ is constant. Let $f_{n}: X \rightarrow Y$ and $f: X \rightarrow Y$ be holomorphic maps, where $X$ is a Banach analytic manifold.

Assume that $A$ is a complex hypersurface in $X$ such that the sequence $\left\{f_{n}\right\}_{n=1}^{\infty}$ converges to $f$ uniformly on compact subsets of $X \backslash A$.

Then the sequence $\left\{f_{n}\right\}_{n=1}^{\infty}$ converges to $f$ uniformly on compact subsets of $X$.

Theorem B. Let $Z$ be a hyperbolically imbedded Banach analytic subspace of a Banach analytic space $Y$ and let $A$ be a complex hypersurface of a Banach analytic manifold $X$ with only normal crossings.

Let $f_{n}: X \rightarrow Y$ and $f: X \rightarrow Y$ be holomorphic maps such that $f_{n}(X \backslash A) \subset Z$ and $f(X \backslash A) \subset Z$.

Assume that the sequence $\left\{f_{n}\right\}_{n=1}^{\infty}$ converges to $f$ uniformly on compact subsets of $X \backslash A$.

Then the sequence $\left\{f_{n}\right\}_{n=1}^{\infty}$ converges to $f$ uniformly on compact subsets of $X$.

Theorem B in the finite dimensional case was first proved by Noguchi [No] with a difficult proof. More recently, Joseph and Kwack [J-K] gave a simpler proof of this theorem. Our proof for Theorem B was based on the ideas of Joseph and Kwack.

We would like to emphasize here that the local compactness of (finite dimensional) complex manifolds plays an essential role in proving the finite dimensional Noguchi theorem. Since Banach analytic manifolds do not have the local compactness property, the technique for proving the Noguchi theorem in the infinite dimensional case required substantial changes. The proofs of the above-mentioned theorems are based on the maximum principle for plurisubharmonic functions.

\section{Preliminary Definitions}

1. We shall make use of properties of Banach analytic spaces as in the books of Ramis [Ra] and Mazet [Ma], and properties of the Kobayashi pseudo-distance on Banach analytic spaces as in Vesentini-Franzoni $[\mathrm{V}-\mathrm{F}]$ or Vesentini $[\mathrm{Ve}$.

2. Let $Y$ be a Banach analytic space and $Z$ a hyperbolic analytic subspace of $Y$. Let $d_{Z}$ denote the Kobayashi distance of $Z$.

We say that $Z$ is hyperbolically imbedded in $Y$ if $\left\{p_{n}\right\}$ and $\left\{q_{n}\right\}$ are sequences in $Z$ such that $\left\{p_{n}\right\} \rightarrow p$ and $\lim _{n \rightarrow \infty} d_{Z}\left(p_{n}, q_{n}\right)=0$, then $\left\{q_{n}\right\} \rightarrow p$.

3. Let $A$ be a complex hypersurface of a Banach analytic manifold $X$. We say that $A$ has normal crossings if, locally, $X \backslash A=\Delta^{* n} \times D$, where $\Delta^{*}$ is the punctured disc in $\mathbf{C}$ and $D$ is an open subset in a Banach space $B$.

4. For every $r>0$, we put $\Delta_{r}=\{z \in \mathbf{C} ;|z|<r\}, \Delta_{r}^{*}=\Delta_{r} \backslash\{0\}$ and $\bar{\Delta}_{r}=\{z \in$ $\mathbf{C} ;|z| \leq r\}$. In particular, $\Delta_{1}=\Delta, \Delta_{1}^{*}=\Delta^{*}$ and $\bar{\Delta}_{1}=\bar{\Delta}$.

5. For every $0<r<s$, we put $\Delta_{r, s}=\{z \in \mathbf{C} ; r<|z|<s\}$ and $\bar{\Delta}_{r, s}=\{z \in$ $\mathbf{C} ; r \leq|z| \leq s\}$.

6. A Banach analytic manifold is said to be weakly disc-convex (see $[\mathrm{T}-\mathrm{H}]$ ) if every sequence $\left\{f_{n}\right\} \subset H(\Delta, X)$ converges in $H(\Delta, X)$ whenever the sequence $\left\{\left.f_{n}\right|_{\Delta^{*}}\right\} \subset H\left(\Delta^{*}, X\right)$ converges in $H\left(\Delta^{*}, X\right)$. Here, denote $H(X, Y)$ as the space of holomorphic mappings from a Banach analytic space $X$ into a Banach analytic space $Y$ equipped with the compact-open topology. 


\section{Proof of Theorem A}

In order to prove Theorem A we need the following:

Lemma (see [T-H, Theorem 2.3]). Let X be a pseudoconvex Banach manifold having $C^{1}$-partitions of unity. Suppose that every holomorphic map $\varphi: \mathbf{C} \rightarrow X$ with $\varphi(\mathbf{C}) \subset \subset X$ is constant.

Then $X$ is disc-convex.

(i) First of all, assume that $A$ is a nonsingular complex hypersurface in $X$. By localizing we may assume that $X=\Delta \times V$ and $A=\{0\} \times V$ and $X \backslash A=\Delta^{*} \times V$.

Let $\left\{\left(z_{k}^{\prime}, z_{k}\right)\right\} \subset \Delta \times V$ be any sequence converging to $\left(z_{0}^{\prime}, z_{0}\right) \in \Delta \times V$. We now prove the sequence $\left\{f_{k}\left(z_{k}^{\prime}, z_{k}\right)\right\} \rightarrow f_{0}\left(z_{0}^{\prime}, z_{0}\right)$.

Indeed, for each $k \geq 0$, consider the holomorphic mapping $\varphi_{k}: \Delta \rightarrow Y$ given by $\varphi_{k}(z)=f_{k}\left(z, z_{k}\right)$ for all $z \in \Delta$. Then $\left.\left\{\left.\varphi_{k}\right|_{\Delta^{*}}\right\} \rightarrow \varphi_{0}\right|_{\Delta^{*}}$ in $H\left(\Delta^{*}, Y\right)$. Since $Y$ is weakly disc-convex, we have $\left\{\varphi_{k}\right\} \rightarrow \varphi_{0}$ in $H(\Delta, Y)$. Thus $\left\{\varphi_{k}\left(z_{k}^{\prime}\right)=f_{k}\left(z_{k}^{\prime}, z_{k}\right)\right\} \rightarrow$ $\varphi_{0}\left(z_{0}^{\prime}\right)=f_{0}\left(z_{0}^{\prime}, z_{0}\right)$.

(ii) Now let $A$ be an arbitrary complex hypersurface in $X$.

Let $\alpha \in A$ be an arbitrary point of $A$. By a theorem of Ramis [Ra, Corollaire, p. 14], there exists a neighbourhood $U$ of $\alpha$ in $X$ which is isomorphic to a neighbourhood $V \times \Delta e \cong V \times \Delta$ of 0 in a Banach space $B$ and a Weierstrass polynomial $P(x, \lambda)=\lambda^{p}+a_{p-1}(x) \cdot \lambda^{p-1}+\ldots+a_{0}(x)$ such that $U \cap \operatorname{Zero}(P)=U \cap A$, for some decomposition $B=E \oplus \mathbf{C} e \cong E \oplus \mathbf{C}$ of $B$.

We have $\operatorname{Zero}(P)=\left(Z \operatorname{Zero}(P) \backslash Z \operatorname{Zero}\left(\frac{\partial P}{\partial \lambda}\right)\right) \cup\left(Z \operatorname{ero}(P) \cap Z \operatorname{Zero}\left(\frac{\partial P}{\partial \lambda}\right)\right)$.

By (i), the sequence $\left\{f_{n}\right\}$ converges uniformly to $f$ on compact subsets of $U \backslash$ $\operatorname{Zero}\left(\frac{\partial P}{\partial \lambda}\right)$.

Repeating this process the sequence $\left\{f_{n}\right\}$ converges uniformly to $f$ on compact subsets of $U \backslash Z \operatorname{Zero}\left(\frac{\partial^{2} P}{\partial^{2} \lambda}\right), \ldots, U \backslash Z \operatorname{ero}\left(\frac{\partial^{p} P}{\partial^{p} \lambda}\right)=U$.

\section{Proof of Theorem B}

Since the problem is local, without loss of generality we may assume that $X=$ $\Delta^{n} \times D, X \backslash A=\Delta^{* n} \times D$, where $D$ is an open subset in a Banach space $B$.

(i) Put $D_{1}=\Delta^{* n-1} \times D$ and fix an arbitrary point $z_{0} \in D_{1}$. Choose open neighbourhoods $W_{1}, W_{2}$ of $b=f\left(0, z_{0}\right)$ in $Y$ such that $W_{1}$ is isomorphic to an analytic subset of an open ball of a Banach space $\widetilde{B}$ and $\bar{W}_{2} \subset W_{1}$.

We now prove that there exist a neighbourhood $V$ of $z_{0}$ in $D_{1}$, a positive number $r>0$ and a positive integer $n_{0}$ such that

$$
f_{n}\left(\Delta_{r}^{*} \times V\right) \subset W_{2} \text { for every } n \geq n_{0} .
$$

Suppose on the contrary that $(*)$ does not hold. Then there exist $\left\{n_{k}\right\}_{k=1}^{\infty} \uparrow \infty$ and $\left\{z_{k}\right\}_{k=1}^{\infty} \rightarrow z_{0}$ such that $f_{n_{k}}\left(\Delta_{\frac{1}{k}}^{*} \times\left\{z_{k}\right\}\right) \not \subset W_{2}$ for all $k \geq 1$.

For each $n \geq 1$, we put

$$
T_{n}=\overline{\left\{z_{k} ; k \geq n\right\}} \text { and } Z_{n}=f\left(\bar{\Delta}_{\frac{1}{n}} \times T_{n}\right) .
$$

Since $\bigcap_{n=3}^{\infty} Z_{n}=\{b\}$, there exists a sequence of open neighbourhoods $\left\{W_{n}\right\}_{n=3}^{\infty}$ of $b$ in $Y$ such that $Z_{n} \subset W_{n}$ for every $n \geq 3$ and

$$
\bigcap_{n=3}^{\infty} W_{n}=\{b\} .
$$


Without loss of generality we may assume that $W_{n} \subset W_{2}$ for all $n \geq 3$.

We now construct inductively $\left\{m_{j}\right\}_{j=3}^{\infty} \uparrow,\left\{r_{j}\right\}_{j=3}^{\infty},\left\{s_{j}\right\}_{j=3}^{\infty}$ such that, for each $j \geq 3$, the following are satisfied:

+) $0<s_{j}<r_{j}<\frac{1}{j}$,
$+) f_{m_{j}}\left(\partial \Delta_{r_{j}} \times T_{j}\right) \not \subset W_{2}$,
$+) f_{m_{j}}\left(\left(\partial \Delta_{s_{j}} \times T_{j}\right) \cup\left(\partial \Delta_{\frac{1}{j}} \times T_{j}\right)\right) \subset W_{j}$,
$+) f_{m_{j}}\left(\bar{\Delta}_{s_{j}, \frac{1}{j}} \times T_{j}\right) \subset W_{1}$.

Assume that $j=3$.

Fix $0<\tilde{r}_{3}<\frac{1}{3}$. Since $f\left(\bar{\Delta}_{\tilde{r}_{3}, \frac{1}{3}} \times T_{3}\right) \subset W_{3}$ and $\left\{f_{n}\right\}$ converges to $f$ uniformly on $\bar{\Delta}_{\tilde{r}_{3}, \frac{1}{3}} \times T_{3}$, it implies that there exists $n_{3} \geq 1$ such that $f_{n}\left(\bar{\Delta}_{\tilde{r}_{3}, \frac{1}{3}} \times T_{3}\right) \subset W_{3}$ for all $n \geq n_{3}$.

Put $r_{3}=\inf \left\{0<r<\frac{1}{3} ; f_{n}\left(\left\{z \in \Delta ; r<|z| \leq \frac{1}{3}\right\} \times T_{3}\right) \subset W_{2}\right.$ for all $\left.n \geq n_{3}\right\}$. Since $f_{n_{k}}\left(\Delta_{\frac{1}{k}}^{*} \times\left\{z_{k}\right\}\right) \not \subset W_{2}$ for all $k \geq 1$, it is easy to see that $r_{3}>0$.

Suppose that $f_{n}\left(\bar{\Delta}_{r_{3}, \frac{1}{3}} \times T_{3}\right) \subset W_{2}$ for all $n \geq n_{3}$.

Fix $0<r_{3}^{\prime}<r_{3}$. Since $f\left(\bar{\Delta}_{r_{3}^{\prime}, \frac{1}{3}} \times T_{3}\right) \subset W_{2}$, there exists $n_{3}^{\prime} \geq n_{3}$ such that $f_{n}\left(\bar{\Delta}_{r_{3}^{\prime}, \frac{1}{3}} \times T_{3}\right) \subset W_{2}$ for all $n \geq n_{3}^{\prime}$. This implies that there is $0<r_{3}^{\prime \prime}<r_{3}$ such that, for each $n \geq n_{3}, f_{n}\left(\bar{\Delta}_{r_{3}^{\prime \prime}, \frac{1}{3}} \times T_{3}\right) \subset W_{2}$. This contradicts the definition of $r_{3}$. Thus there exists $m_{3} \geq n_{3}$ such that $f_{m_{3}}\left(\partial \Delta_{r_{3}} \times T_{3}\right) \not \subset W_{2}$.

Since $f_{m_{3}}\left(\bar{\Delta}_{r_{3}, \frac{1}{3}} \times T_{3}\right) \subset \bar{W}_{2} \subset W_{1}$, there exists $0<s_{3}<r_{3}$ such that $f_{m_{3}}\left(\bar{\Delta}_{s_{3}, \frac{1}{3}} \times T_{3}\right) \subset W_{1}$. Moreover, we have $f_{m_{3}}\left(\left(\partial \Delta_{s_{3}} \times T_{3}\right) \cup\left(\partial \Delta_{\frac{1}{3}} \times T_{3}\right)\right) \subset W_{3}$.

Now let $j \geq 4$ be given.

Fix $0<\tilde{r}_{j}<\frac{1}{j}$. Since $f\left(\bar{\Delta}_{\tilde{r}_{j}, \frac{1}{j}} \times T_{j}\right) \subset W_{j}$ and $\left\{f_{n}\right\}$ converges to $f$ uniformly on $\bar{\Delta}_{\tilde{r}_{j}, \frac{1}{j}} \times T_{j}$, it implies that there exists $n_{j}>m_{j-1}$ such that $f_{n}\left(\bar{\Delta}_{\tilde{r}_{j}, \frac{1}{j}} \times T_{j}\right) \subset W_{j}$ for all $n \geq n_{j}$.

Put $r_{j}=\inf \left\{0<r<\frac{1}{j} ; f_{n}\left(\left\{z \in \Delta ; r<|z| \leq \frac{1}{j}\right\} \times T_{j}\right) \subset W_{2}\right.$ for all $\left.n \geq n_{j}\right\}$.

Repeating as above, we have $0<r_{j}<\frac{1}{j}$ and $f_{m_{j}}\left(\partial \Delta_{r_{j}} \times T_{j}\right) \not \subset W_{2}$ for some $m_{j} \geq n_{j}$ and $f_{m_{j}}\left(\bar{\Delta}_{s_{j}, \frac{1}{j}} \times T_{j}\right) \subset W_{1}$ for some $0<s_{j}<r_{j}$. Moreover,

$$
f_{m_{j}}\left(\left(\partial \Delta_{s_{j}} \times T_{j}\right) \cup\left(\partial \Delta_{\frac{1}{j}} \times T_{j}\right)\right) \subset W_{j}
$$

We now put

$$
K=\bigcup_{j=3}^{\infty} f_{m_{j}}\left(\left(\partial \Delta_{s_{j}} \times T_{j}\right) \cup\left(\partial \Delta_{\frac{1}{j}} \times T_{j}\right)\right)
$$

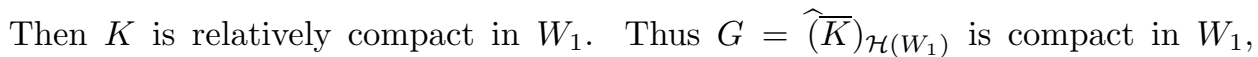
where $\mathcal{H}\left(W_{1}\right)$ is the set of all holomorphic functions on $W_{1}$. Since $\partial \Delta_{r_{j}} \times T_{j} \subset$ $\Delta_{s_{j}, \frac{1}{j}} \times T_{j}$, by the maximum principle, it follows that $f_{m_{j}}\left(\partial \Delta_{r_{j}} \times T_{j}\right) \subset G$ for all $j \geq 1$. Since $f_{m_{j}}\left(\partial \Delta_{r_{j}} \times T_{j}\right) \not \subset W_{2}$, there exist $\lambda_{j} \in \partial \Delta_{r_{j}}$ and $z_{k_{j}} \in T_{j}$ such that $f_{m_{j}}\left(\lambda_{j}, z_{k_{j}}\right) \in \partial W_{2}$. By the compactness of $G$, without loss of generality we may assume that $\left\{f_{m_{j}}\left(\lambda_{j}, z_{k_{j}}\right)\right\} \rightarrow x_{1} \in \partial W_{2}$. Since $d_{\Delta^{*}}\left(\partial \Delta_{r_{j}}\right) \rightarrow 0$ and by the distance 
decreasing principle, it follows that $d_{Z}\left(f_{m_{j}}\left(\partial \Delta_{r_{j}} \times\left\{z_{k_{j}}\right\}\right)\right) \rightarrow 0$ as $j \rightarrow \infty$. Since $Z$ is hyperbolically imbedded into $Y$, we have $f_{m_{j}}\left(\partial \Delta_{r_{j}} \times\left\{z_{k_{j}}\right\}\right) \rightarrow x_{1}$ as $j \rightarrow \infty$.

On the other hand, since $f_{m_{j}}\left(\left(\partial \Delta_{s_{j}} \times T_{j}\right) \cup\left(\partial \Delta_{\frac{1}{j}} \times T_{j}\right)\right) \subset W_{j}$ and $\bigcap_{j=3}^{\infty} W_{j}=\{b\}$, it follows that $f_{m_{j}}\left(\partial \Delta_{s_{j}} \times\left\{z_{k_{j}}\right\}\right) \rightarrow b$ and $f_{m_{j}}\left(\partial \Delta_{\frac{1}{j}} \times\left\{z_{k_{j}}\right\}\right) \rightarrow b$ as $j \rightarrow \infty$.

Choose a continuous linear functional $u$ on $\widetilde{B}$ such that $u\left(x_{1}\right) \neq u(b)$. Consider the holomorphic function $\sigma_{j}: \bar{\Delta}_{s_{j}, \frac{1}{j}} \rightarrow \mathbf{C}$ given by $\sigma_{j}(\lambda)=u \circ f_{m_{j}}\left(\lambda, z_{k_{j}}\right)$ for every $\lambda \in \bar{\Delta}_{s_{j}, \frac{1}{j}}$. Since $\left\{\sigma_{j}\left(\partial \Delta_{s_{j}}\right)\right\} \rightarrow u(b)$ and $\left\{\sigma_{j}\left(\partial \Delta_{\frac{1}{j}}\right)\right\} \rightarrow u(b)$, we have

$$
\begin{array}{r}
\forall \varepsilon>0, \exists N, \forall j \geq N, \forall \theta:\left|\sigma_{j}\left(\frac{1}{j} e^{i \theta}\right)-u(b)\right|<\varepsilon \text { and } \\
\left|\sigma_{j}\left(s_{j} e^{i \theta}\right)-u(b)\right|<\varepsilon .
\end{array}
$$

Applying the maximum principle to the function $z \mapsto \sigma_{j}(z)-u(b)$ on the annulus $\Delta_{s_{j}, \frac{1}{j}}$, in particular the circle $\partial \Delta_{r_{j}} \subset \Delta_{s_{j}, \frac{1}{j}}$, it implies that $\left|\sigma_{j}\left(r_{j} e^{i \theta}\right)-u(b)\right|<\varepsilon$ for every $\theta$. Thus $u(b)=u\left(x_{1}\right)$. This is impossible. Therefore the assertion $(*)$ is proved.

From $(*)$ we deduce immediately that $f_{n}\left(\Delta_{r} \times V\right) \subset W_{1}$ for every $n \geq n_{0}$ and $f\left(\Delta_{r} \times V\right) \subset W_{1}$.

By the maximum principle it follows that $\left\{f_{n}\right\}$ converges uniformly to $f$ on compact subsets in $\Delta \times \Delta^{* n-1} \times D$.

(ii) Repeating this process the sequence $\left\{f_{n}\right\}$ converges uniformly to $f$ on compact subsets of $\Delta^{2} \times \Delta^{* n-2} \times D, \Delta^{3} \times \Delta^{* n-3} \times D, \ldots, \Delta^{n} \times D=X$.

\section{ACKNOWLEDGEMENTS}

The authors would like to thank the referee whose suggestions and corrections improved this paper. We would also like to thank Professor N.V. Khue for his comments.

\section{REFERENCES}

[J-K] J.E. Joseph and M.H. Kwack, Hyperbolic embedding and spaces of continuous extensions of holomorphic maps, J. Geom. Analysis 4(1994), 361-378. MR 95g:32037

[Ko] S.Kobayashi, Hyperbolic Complex Spaces, vol. 318(1998), Grundlehren der mathematischen Wissenschaften. MR 99m:32026

[La] S. Lang, Introduction to Complex Hyperbolic Spaces, Springer-Verlag (1987). MR 88f:32065

[Ma] P. Mazet, Analytic Sets in Locally Convex Spaces, Math. Studies, North-Holland, v.121(1987). 1984 edition MR 86i:32012

[Mu] J. Mujica, Complex Analysis in Banach Spaces, Math. Studies, North-Holland, v.120(1986). MR 88d:46084

[No] J. Noguchi, Moduli spaces of holomorphic mappings into hyperbolically imbedded complex spaces and locally symmetric spaces, Invent. Math. 93(1988), 15-34. MR 89j:32031

[N-O] J. Noguchi and T. Ochiai, Geometric Function Theory in Several Complex Variables, Translations of Math. Monographs, Amer. Math. Soc., v. 80(1990). MR 92e:32001

[Ra] J. P. Ramis, Sous-ensembles Analytiques d'une Variété Banachique Complexe, SpringerVerlag (1970). MR 45:2205

[T-H] Do Duc Thai and Nguyen Le Huong, On the disc-convexity of Banach analytic manifolds, Ann. Pol. Math. 69(1998), 1-11. MR 2000b:32042 
[V-F] E. Vesentini and T. Franzoni, Holomorphic Maps and Invariant Distances, Math. Studies, North-Holland, v. 40(1980). MR 82a:32032

[Ve] E. Vesentini, Invariant distances and invariant differential metric in locally convex spaces, Spectral theory, Banach Centre Publication U.8 P.W.N, Polish Sci. Publisher Warsaw, (1982), 493-512. MR 85d:32049

Department of Mathematics, Pedagogical University of Hanoi, Caugiay, Hanoi, VietNAM

E-mail address: ddthai@netnam.org.vn

Current address: Department of Mathematics, Hanoi University of Education, Cau Giay, Tu Liem, Hanoi, Vietnam

Department of Mathematics, Pedagogical University of Vinh, Vinh, Vietnam 